\title{
DIFFERENTIAL EFFECTS OF FOCAL AND AMBIENT VISUAL PROCESSING DEMANDS ON DRIVING PERFORMANCE
}

\author{
John K. Lenneman, Joseph Lenneman, Nicholas Cassavaugh \& Richard Backs \\ Central Michigan University \\ Mount Pleasant, MI, USA \\ Email:johnlenneman@cablespeed.com
}

\begin{abstract}
Summary: In this study, the differential effects of focal and ambient visual demand on driving were investigated. Subjects participated in a dual-task experiment in which they performed a driving simulation task and a focal or ambient side-task. It was predicted that the focal side-task would cause a significant deterioration in the maintenance of longitudinal control but not lateral control, while there should be no effects of the ambient side-task on driving performance. In general, the results suggest a differentiation in the processing demands of focal and ambient vision.
\end{abstract}

\section{INTRODUCTION}

Recent modifications to multiple resource theory detail a new dichotomy of visual attention channels: focal visual processes (required for fine detail and pattern recognition) and ambient visual processes (required for sensing orientation and egomotion; Wickens, 2002). There is evidence that suggests resources available to process information in focal vision are different from the resources available to process information in ambient vision (Leibowitz \& Post, 1982). Further, it has been suggested that the processing of ambient visual information is pre-attentive, implying that it does not demand attention (Previc, 1998; Wickens, 2002). Finally, while peripheral and ambient vision are highly correlated, they are not two terms for the same concept. The egomotion processes that characterize ambient vision can be processed in the foveal region as well as deep into the periphery with very little degradation of detection. Thus, while focal vision is tightly coupled to the fovea, ambient vision is both foveal and peripheral.

Research suggests that, in general, ambient vision is used to maintain vehicle control (e.g., lane keeping) whereas focal vision is used to maintain headway (the distance between your vehicle and the vehicle in front of you) and respond to hazard events (Summala, Nieminen, and Punto, 1996; Summala, Lamble, and Lasko, 1998). Through a series of studies, Horrey, Wickens, and Consalas (2006) were able to demonstrate that performing a side-task that draws upon focal visual attention resources while driving results in the deterioration of the focal component of a driving task (hazard response), but not in the ambient component (lane keeping). The purpose of the current study was to investigate the differentiation of focal and ambient visual processing attentional demands on driving performance in a dual-task driving environment.

Subjects participated in a dual-task driving simulation that contained focal and ambient components for both the driving task and the side-task. Because both longitudinal control of a vehicle (the focal component of vehicular control) and a focal side-task rely on the same visual attention resources, visual attention resource competition should arise causing deterioration in the maintenance of longitudinal control. Further, an increase in difficulty of the focal side-task will cause greater deterioration in the maintenance of longitudinal control. However, if the side- 
task can be performed using ambient visual processes, parallel processing can occur between the tasks of maintaining longitudinal control of the vehicle and side-task performance. Thus, a subject's ability to maintain longitudinal control should not deteriorate. Further, an increase in difficulty of the ambient side-task should not cause an increase in deterioration in the maintenance of longitudinal control.

For lateral control (the ambient component of vehicular control), there should be no resource competition between driving and either of the side-tasks. When the side-task is focal, parallel processing can occur between driving and performing the side-task because lateral control of the vehicle can be maintained using ambient vision. When the side-task is ambient, lateral control of the vehicle should not be affected by the side-task because ambient visual information is processed pre-attentively. Thus, a subject's ability to maintain lateral control (the ambient component) should not deteriorate. Further, an increase in focal and ambient side-task difficulty should not cause deterioration in the maintenance of lateral control.

\section{METHOD}

\section{Subjects}

Sixty four subjects (34 male, 30 female) were recruited through Central Michigan University's Psychology Department subject recruitment pool for participation. Subject's ages ranged from 18 to 42 years $(M=20.70, S D=3.79)$. Administration of the UFOV exam revealed that all subjects had normal central vision, divided attention, and selective attention capabilities.

\section{Driving Simulation Overview}

A desktop DriveSafety driving simulator running HyperDrive software (ver. 1.9) was used to present the driving task. The driving environment consisted of a $20 \mathrm{~km}$ long straight semiindustrial, two-lane bi-directional road with occasional cross streets and parked cars on the side of the roadway (approximately 9-10 per $\mathrm{km}$ ). Subjects were instructed to both steer the simulated vehicle and control velocity by using the brake and acceleration pedals. To facilitate the use of focal vision while driving, subjects were required to follow a lead vehicle and maintain a consistent amount of headway best they could. To facilitate the use of ambient vision, subjects were instructed to keep their vehicle centered in the right lane. Two difficulty levels of headway maintenance and lane keeping were established by varying the velocity of the lead vehicle (more lead vehicle speed variability was more difficult) and wind disturbance (higher wind disturbance levels were more difficult), respectively.

\section{Side-Task Overview}

A second computer was used to present the side-tasks on a 5" diagonal LCD screen mounted directly below the driving simulation monitor. Subjects were positioned so that the visual angle from the center of the primary driving display to the center of the side-task display was approximately $8^{\circ}$. Side-tasks were created that require the use of focal or ambient visual resources. Focal demand was imposed by requiring subjects to determine which side (left or right) of the side-task display a letter was presented. A number was always presented on the 
opposite side of the display (see panels a and b in Figure 1). Subjects were allowed to scan freely between the driving display and side-task display. Two levels of focal side-task difficulty were established by varying the level of stimuli blur. Higher blur was more difficult (see panels a and $\mathrm{b}$ in Figure 1). Ambient demand of the side-task was achieved by asking subjects to determine which of two simulated gauges on the side-task display was increasing. The gauges were placed on the left and right side of the display. Subjects were required to perform the ambient side-task while maintaining foveal vision on the driving display; they were not allowed to look at the sidetask display. Video was reviewed and confirmed that subjects indeed did not look at the side-task display when performing the ambient side-task. Two levels of ambient side-task difficulty were established by varying the rate of increase in the level of the gauge display (lower rate of change was more difficult; in panel c of Figure 1 the right gauge is increasing). Subjects input their response to the side-task by pressing either a left or right button on the steering wheel.
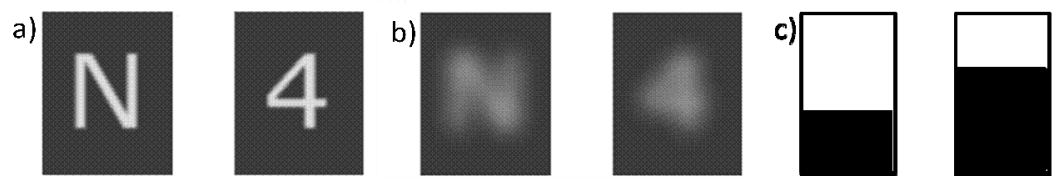

Figure 1. The two side tasks

Visual channel of the side-task served as a between-subjects variable (32 subjects participated in ambient side-tasks, 32 participated in focal side-tasks). A total of 10 stimuli were presented during a $90 \mathrm{~s}$ block. Side-task stimuli were presented at the beginning of every block and every 8-10 s thereafter (the average inter-stimulus interval within a block was always $9 \mathrm{~s}$ ).

\section{Procedure}

Upon arrival subjects filled out a consent form and a biographical questionnaire and were administered a UFOV examination (to ensure that subjects were free of any functional vision or visual attention limitations). Subjects first practiced the driving simulator task twice, which was followed by side-task only practice and finally, dual-task driving with side-task practice. Data collection consisted of three experimental conditions, occurring in the following order: driving only, side-task only, and dual-task driving with side-task performance. In each condition, all possible factorial combinations were presented (wind and lead vehicle speed variability for driving only, side-task difficulty for side-task only, and all three for dual-task driving with sidetask difficulty). Difficulty levels in all conditions were balanced.

\section{Performance Measures}

Physiological data were collected in this study (but not presented in this paper). Performance data were reduced to the same epochs used to compute the physiological measures (a $60 \mathrm{~s}$ buffer epoch and a $30 \mathrm{~s}$ data analysis epoch). Therefore, performance data presented in this paper are from the final $30 \mathrm{~s}$ of the full $90 \mathrm{~s}$ block. Performance on the side-tasks was assessed by measuring response time (from the onset of stimulus presentation to steering wheel button press in $\mathrm{ms}$ ) and accuracy. The measure of lateral control of the vehicle was the root-mean-squared of lateral deviations from lane center (RMS lateral deviation in $\mathrm{m}$ ). The measure of longitudinal 
control was the standard deviation of headway (the deviation between the subject's vehicle and the lead vehicle; SD headway in $\mathrm{m}$ ).

For dual-task analyses, decrement scores were calculated as the difference between the raw score of a dual-task observation and the corresponding raw score of single-task observation for that measure. Positive scores indicate an increase in the measure from single- to dual-task performance (i.e., longer reaction times, increased error in longitudinal and lateral control). Negative scores indicate a decrease in the measure from single- to dual-task performance (i.e., shorter reaction times, decreased error in longitudinal and lateral control). Thus, positive scores indicate a deterioration in performance from single- to dual-task. Negative scores are indicative of an improvement in performance from single- to dual-task.

\section{RESULTS}

All analyses were conducted separately for focal and ambient side-task groups to reflect the structure of the predictions previously described.

\section{Side-task Performance}

Accuracy of side-task only performance was perfect for most subjects. Thus, all analyses were conducted on reaction time (RT) only. To test the effects of the difficulty manipulations, paired $t$-tests were conducted for RT separately for ambient and focal side-task performance. Reaction times increased significantly from low side-task difficulty $(M=1.28 \mathrm{~s}, S D=0.55)$ to high sidetask difficulty $(M=1.92 \mathrm{~s}, S D=0.67)$ when performing the ambient side-task, $t(31)=7.22, p$ $<.001$ and from low side-task difficulty $(M=0.89 \mathrm{~s}, S D=0.84)$ to high side-task difficulty $(M=$ $0.97 \mathrm{~s}, S D=0.73$ ) when performing the focal side-task, $t(31)=2.09, p<.05$.

Analysis of the change (the decrement in reaction time; $\mathrm{RT}_{\mathrm{dec}}$ ) from side-task only to dual-task driving with side-task performance (collapsed across all conditions) revealed that reaction time significantly increased $(M=0.31 \mathrm{~s}, S D=0.41)$ from ambient side-task only to dual-task driving with ambient side-task performance, $F(1,31)=18.32, p<.001$, partial $\eta^{2}=.371$, and increased $(M=0.17 \mathrm{~s}, S D=0.18)$ from focal side-task only to dual-task driving with focal side-task performance, $F(1,31)=28.83, p<.001$, partial $\eta^{2}=.482$.

For dual-task driving with ambient side-task performance, the main effect of side-task difficulty $\left(p=.211\right.$, partial $\left.\eta^{2}=.050\right)$ was not significant. For dual-task driving with focal side-task performance, $\mathrm{RT}_{\text {dec }}$ increased from low side-task difficulty $(M=0.10 \mathrm{~s}, S D=0.20)$ to high sidetask difficulty $(M=0.23 \mathrm{~s}, S D=0.23), F(1,31)=8.79, p<.01$, partial $\eta^{2}=.221$.

\section{Driving Performance}

Analyses of the change in performance from driving-only to dual-task driving with side-task performance were conducted to determine if the addition of the side-task to driving significantly affected driving performance. Tests of the main effect of side-task difficulty were used to determine if the increase in side-task difficulty significantly affected driving performance. Analyses were done separately for the focal and ambient side-task visual channels. 
The Maintenance of Longitudinal Control. It was predicted that dual-task driving with focal side-task performance will cause a deterioration in longitudinal control (an increase in SD headway) compared to driving-only; whereas, dual-task driving with ambient side-task performance will not cause any change in a subject's ability to maintain longitudinal control compared to driving-only. The results revealed an increase in SD headway from driving-only to dual-task driving with focal side-task performance, $F(1,31)=4.51, p<.05$, partial $\eta^{2}=.127$ (see Table 1). There was no change in SD headway from driving-only to dual-task driving with ambient side-task performance $\left(p=.844\right.$, partial $\left.\eta^{2}=.001\right)$. Thus, the results supported the hypothesis. The maintenance of longitudinal control did significantly deteriorate when performing the focal side-task while driving, and not when performing the ambient side-task.

Table 1. Means and standard deviations of performance measures decrement scores for dual-task driving with side-task performance

\begin{tabular}{lcc}
\hline Measure & $\begin{array}{c}\text { Driving with } \\
\text { Focal Side-task }\end{array}$ & $\begin{array}{c}\text { Driving with } \\
\text { Ambient Side-task }\end{array}$ \\
\hline SD Headway (m) & $1.12^{*}$ & 0.12 \\
RMS Lateral & $(0.53)$ & $(0.63)$ \\
Deviation $(\mathrm{m})$ & 0.00 & 0.01 \\
\hline
\end{tabular}

It was also predicted that an increase in focal side-task difficulty would cause deterioration in longitudinal control, whereas there would be no significant effect of the ambient side-task. The results revealed that the change in SD headway from low to high side-task difficulty while performing a focal side-task was not significant $\left(p=.448\right.$, partial $\left.\eta^{2}=.019\right)$, whereas, SD headway decreased significantly from low to high side-task difficulty while performing the ambient side-task, $F(1,31)=4.20, p<.05$, partial $\eta^{2}=.119($ see Table 2$)$. Although there was a differential effect of side-task visual channel when side-task difficulty was increased, it was not as predicted (deterioration in focal performance was predicted, whereas performance improved when performing the ambient side-task). Because of the unexpected effect of low difficulty ambient side-task performance, this hypothesis was not supported.

Table 2. Means and standard deviations of performance measures decrement scores for dual-task driving with low and driving with high side-task difficulty

\begin{tabular}{lcccc}
\hline & \multicolumn{2}{c}{ Focal } & \multicolumn{2}{c}{ Ambient } \\
\cline { 2 - 5 } Measure & $\begin{array}{c}\text { Driving with Low } \\
\text { Side-task Difficulty }\end{array}$ & $\begin{array}{c}\text { Driving with High } \\
\text { Side-task Difficulty }\end{array}$ & $\begin{array}{c}\text { Driving with Low } \\
\text { Side-task Difficulty }\end{array}$ & $\begin{array}{c}\text { Driving with High } \\
\text { Side-task Difficulty }\end{array}$ \\
\hline \multirow{2}{*}{ SD Headway (m) } & 0.94 & 1.30 & 0.74 & -0.49 \\
& $(0.57)$ & $(0.58)$ & $(0.84)$ & $(0.54)$ \\
RMS Lateral & 0.00 & 0.01 & 0.02 & 0.00 \\
Deviation (m) & $(0.01)$ & $(0.02)$ & $(0.01)$ & $(0.01)$ \\
\hline
\end{tabular}

$* \mathrm{p}<.05, * * \mathrm{p}<.01, * * * \mathrm{p}<.001$

The Maintenance of Lateral Control. It was predicted that neither dual-task driving with focal nor ambient side-task performance would cause a deterioration in the maintenance of lateral control (there would be no significant change in RMS lateral deviation) compared to driving- 
only. The results revealed that there was no change in RMS lateral deviation when comparing driving-only to dual-task driving with focal side-task performance $\left(p=.796\right.$, partial $\left.\eta^{2}=.002\right)$ and dual-task driving with ambient side-task performance $\left(p=.406\right.$, partial $\eta^{2}=.022$; see Table 1). Results supported the hypothesis. There was no effect of either side-task on the maintenance of lateral control. To note, the absence of deterioration in lateral control when the side-task was focal could have been because of parallel processing between the focal side-task and ambient component of the driving task (lane keeping). However, when the side-task was ambient, maintaining lateral control was predicted to have been because of the pre-attentiveness of ambient visual information. Resource theories suggest that if ambient visual information requires attention to be processed, then there would have been resource conflict which, in turn, would have manifested in performance as a deterioration of lateral control; which of course, it did not.

It was also predicted that an increase in focal or ambient side-task difficulty would not have any effects on the maintenance of lateral control (no significant change in RMS lateral deviation). The results revealed that there was no change in RMS lateral deviation when comparing dualtask driving with low to high side-task difficulty for the focal $\left(p=.649\right.$, partial $\left.\eta^{2}=.007\right)$ and ambient $\left(p=.056\right.$, partial $\left.\eta^{2}=.113\right)$ side-task conditions (see Table 2$)$. The results supported the hypothesis. There was no effect of an increase in side-task difficulty of the focal or the ambient side-task on the maintenance of lateral control.

\section{DISCUSSION}

Our results provide support for the notion that ambient visual information can be processed preattentively. In the current study, we found that dual-task driving and performance of the focal secondary task caused deterioration in driving performance that was not observed when the secondary task was ambient (recall that there was no effect of the ambient side-task on the SD headway when collapsed across side-task difficulty). Similarly, Weinstein and Wickens (1992) found that peripheral tasks drawing upon focal visual resources deteriorated dual-task performance more than peripheral tasks that draw upon ambient resources. Further, the results of the current study also revealed that performance of a focal side-task while driving caused a deterioration in the focal component of driving (the maintenance of headway), but not in the ambient component (the maintenance of lateral control). These results are consistent the findings of Horrey et al. (2006) and Summala and his colleagues (Summala et al., 1996; Summala et al., 1998; Lamble et al., 2002) which demonstrated that concurrent driving and secondary task performance can cause deterioration in the focal component of driving (e.g., hazard detection, headway maintenance) and not the ambient component (e.g., lane keeping).

However, not all of the results provided support for the notion that ambient visual information can be processed pre-attentively. For example, the significant deterioration in ambient side-task performance from side-task only to dual-task driving with ambient side-task performance represents a cost of concurrence during dual-task performance. If ambient visual information is processed pre-attentively, then there should not have been any significant effects on side-task performance. Further, it was predicted that an increase in ambient side-task difficulty would have no effect on the maintenance of longitudinal control if ambient visual information could be processed pre-attentively. Not only was this hypothesis not supported, but the results revealed that an increase in ambient side-task difficulty unexpectedly led to a decrease in SD headway. 
However, it is important to note that while the change from low to high difficulty was significant, follow-up analyses revealed that neither condition was significantly different from driving only.

One area of future research could include an investigation of the causes for the decrease in SD headway as ambient side-task difficulty increased. Other areas of future research include the effects of differences in visual attention capabilities, side-task display location, and the type of driving environment. In the current study a simple desktop simulator was used for the driving task. One may question whether the results would have been different if the study was conducted in a larger, more visually rich, driving environment. However, it is important to note that the study conducted by Horrey et al. (2006) was conducted in a simulator that subtended a total of $270^{\circ}$ of visual angle. Thus, we believe that similar results would be found if the same experiment was conducted with a larger, higher fidelity simulator.

Finally, it should be recognized that a number of the conclusions are the result of accepting the null hypothesis. One may argue that the process of accepting the null hypothesis to draw conclusions minimizes some of the claims. However, we believe that in the current study the ability to predict patterns of performance across a set of hypotheses provides good evidence of the pre-attentiveness of ambient visual information.

\section{REFERENCES}

Horrey, W.J., Wickens, C.D., \& Consalus, K.P. (2006). Modeling drivers visual attention allocation while interacting with in-vehicle technologies. Journal of Experimental Psychology: Applied, 12, 67-78.

Lamble, D., Summala, H., \& Hyvarinen, L. (2002). Driving performance of drives with impaired central visual field acuity. Accident Analysis and Prevention, 34, 711-716.

Leibowitz, H.M., \& Post, R.B. (1982). The modes of processing concept and some implications. In J. Beck (Ed.), Organization and Representation in Perception (pp. 343-363). Hillsdale, NJ: Lawrence Erlbaum.

Previc, F.H. (1998). The neuropsychology of 3-D space. Psychological Bulletin, 124, 123-164.

Summala, H., Nieminen, T., \& Punto, M. (1996). Maintaining lane position with peripheral vision during in-vehicle tasks. Human Factors, 38, 442-451.

Summala, H., Lamble, D., \& Lasko, M. (1998). Driving experience and perception of the lead car's braking when looking at in-car targets. Accident Analysis \& Prevention, 30, 401-407

Weinstein, L.F., \& Wickens, C.D. (1992). Use of Nontraditional Flight Displays for the Reduction of Central Visual Overload in the Cockpit. The International Journal of Aviation Psychology, 2, 121-142.

Wickens, C.D. (2002). Multiple resources and performance prediction. Theoretical Issues in Ergonomics Sciences, 3, 159-177. 